\title{
To Assess Association of Oral Habits and Temporomandibular Joint Disorders in Adolescents: A Cohort Study
}

\author{
Dr Shivayogi Charantimath ${ }^{1}$, Dr Abhishek Roy ${ }^{2}$ \\ ${ }^{I}$ Reader, Department of Oral Medicine and Radiology, K.L.E.V.K.Institute of Dental Science, Belgaum \\ ${ }^{2}$ Intern, K.L.E.V.K. Institute of Dental Science
}

\begin{abstract}
Introduction: The etiological factor behind degenerative changes in the temporomandibular joints (TMJs) is controversial. Tooth attrition, improper occlusal andhabits are possible causative factors. Oral habits are common and generally do not harm the stomatognathic apparatus but, when the activity exceeds the individual's physiologic tolerance, the system begins to alter. Although psychological factors are an important etiological component in producing and perpetuating TMJ disorders a comprehensive view of the problem can be understood through the concept of multifactorial etiology. This study aims at assessing the association of oral habits and temporomandibular joint disorders through history of oral habits and clinical examination.

Methodology: Adolescents aged between 15 \& 20 yrs were included in the study. Each participant answered the questioner regarding the oral habits and symptoms of TMD. Oral habits - Chewing sticky products, Bruxism, Biting habits,continuous leaning of the chin on the hand,Clenching were included and recorded. Questionnaire on oral habitsand clinical examination check list were recorded on the basis of RDC Criteria .

Results and observation: The results obtained were analyzed by Chi-Squared test.The analysis of the 30 questionnaires gave insight into the association of oral habits with both sexes. Also, prevalence of various TMD with different habits was seen.This study indicates that there was higher rate of oral habits among women. Men with oral habits have more frequency of mild tmj disorders. Chewing sticky products: 6 males and 4 females had habit of chewing sticky products. Out of 6 male 4 patient had TMJ pain and 2 female had TMJ pain. I male had TMJ pain associated with clicking and jaw lock.Bruxism: 5 males and 3 females reported with bruxism habit. 3 male had TMJ pain and clicking..Imale patient had TMJ pain and 2 female had TMJ pain associated with clicking and jaw lock. Clenching: out of 30 patient 1 male and 2 female reported with clenching. Males didn't report with TMJ pain and clicking and jaw lock. 2 females had TMJ pain associated with clicking and jaw lock.Chewing sticky products +clenching: 3 males and 5 females had habit of chewing sticky products and clenching. 3 males and 1 female reported with TMJ pain. 1 female patient had TMJ pain with clicking and no males had TMJ pain with clicking. 3 females had TMJ pain associated with clicking and jaw lock.Bruxism + clenching: 1 female patient had bruxism and clenching and associated with TMJ pain.Headache/migraine: 9 males were affected with headache and migraine in last 6 months. 13 females were affected with headache and migraine in last 6 months.
\end{abstract}

\section{Introduction}

Modernization has lead to development of many oral habits that may lead to or facial disturbances and temporomandibular disorders.The etiological factor behind degenerative changes in the temporomandibular joints (TMJs) is controversial. Tooth attrition, improper occlusal and habits are possible causative factors. Oral habits are common and generally do not harm the stomatognathic apparatus but, when the activity exceeds the individual's physiologic tolerance, the system begins to alter. Although psychological factors are an important etiological component in producing and perpetuating TMJ disorders a comprehensive view of the problem can be understood through the concept of multifactorial etiology.

This study aims at assessing the association of oral habits and temporomandibular joint disorders through history of oral habits and clinical examination. The purpose of this study is to find a relation between such habits and TMJ difficulties. Previous studies on this topic have mainly concentrated upon the middle age group of adults. But in this study the target age group is of adolescents because they are a group which has a high association rate with such habits. The main objective of this study is to ascertain the adverse effects of such habits to the stomatognathic system and possible gender association

\section{Materials and Methods}

This study wascarried out in Department of Oral Medicine and Radiology K.L.E. V.K .Institute of Dental Sciences Belgaum. The study included 30 adolescents. The patients were examined for signs and symptoms of TMJ. The ethical committee clearance was obtained. The consent form was obtained from patients. Each participantanswered the questioner regarding the oral habits and symptoms of TMD. 
Study tool:

- Questionnaire on oral habits [ RDC CRITERIA] ${ }^{[4,5]}$

- Clinical examination check list [RDC CRITERIA]

Inclusion criteria:Adolescents aged between 15 \& $20 \mathrm{yrs}$

- Oral habits - Chewing stickyproducts, Bruxism, Biting habits, Continuous leaning of the chin on the hand, Clenching.

Exclusion criteria: Patients not willing to participate in study, Patients without oral habits.

\section{Results and Observation}

The results obtained wereanalyzed by Chi-Squared test.The analysis of the 30 questionnaires gave insight into the association of oral habits with both sexes. Also, prevalence of various TMD with different habits was seen.This study indicates that there was higher rate of oral habits among women. Men with oral habits have more frequency of mild tmjdisorders.

Chewing sticky products: 6 males and 4 females had habit of chewing sticky products. Out of 6 male 4 patient had TMJ pain and 2 female had TMJ pain. I male had TMJ pain associated with clicking and jaw lock.

Bruxism: 5 males and 3 females reported with bruxism habit. 3 male had TMJ pain and clicking..1male patient had TMJ pain and 2 female had TMJ pain associated with clicking and jaw lock.

Clenching: out of 30 patient 1 male and 2 female reported with clenching. Males didn't report with TMJ pain and clicking and jaw lock. 2 females had TMJ pain associated with clicking and jaw lock.

Chewing sticky products +clenching: 3 males and 5 females had habit of chewing sticky products and clenching. 3 males and 1 female reported with TMJ pain. 1 female patient had TMJ pain with clicking and no males had TMJ pain with clicking. 3 females had TMJ pain associated with clicking and jaw lock.

Bruxism + clenching: 1 female patient had bruxism and clenching and associated with TMJ pain.

Headache/migraine: 9 males were affected with headache and migraine in last 6 months. 13 females were affected with headache and migraine in last 6 months.

Table 1 :

\begin{tabular}{|l|l|l|l|l|l|l|l|l|}
\hline Habit & \multicolumn{2}{|l|}{ People having Habit } & \multicolumn{2}{l|}{ TMJ Pain } & \multicolumn{2}{l|}{ TMJ Pain + Clicking } & \multicolumn{2}{l|}{ TMJ Pain + Clicking + Jaw Lock } \\
\hline & Males & Females & Males & Females & Males & Females & Males & females \\
\hline $\begin{array}{l}\text { 1)Chewing sticky } \\
\text { products }\end{array}$ & 6 & 4 & 4 & 2 & 1 & - & 1 & - \\
\hline 2)Bruxism & 5 & 3 & 1 & 1 & 3 & - & 1 & 2 \\
\hline 3) clenching sticky & 1 & 2 & - & - & - & - & 1 & 2 \\
\hline $\begin{array}{l}\text { 4)Chewing } \\
\text { products + Bruxism }\end{array}$ & 5 & 3 & 4 & - & 1 & - & 3 \\
\hline $\begin{array}{l}\text { 5)Bruxism } \\
\text { Clenching }+\end{array}$ & - & 1 & - & 1 & - & - & - & - \\
\hline $\begin{array}{l}\text { Headache/Migraine } \\
\text { in last 6 months }\end{array}$ & 9 & 13 & - & - & - & - & - & - \\
\hline
\end{tabular}

Graph 1 ; Prevalence of Oral Habits

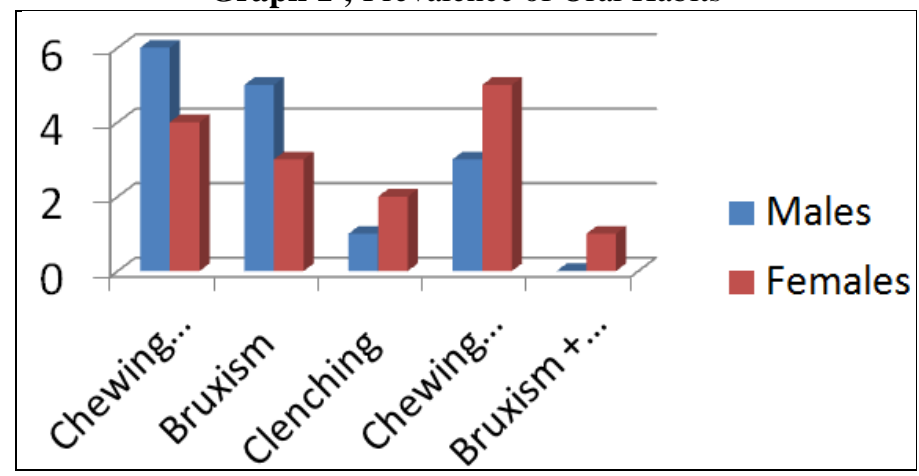

IV. Discussion

This study indicates that there is a higher rate of oral habits amongst women.In women, combination of various oral habits occurs more frequently. Men, with oral habits, have more frequency of mild TMJ disorders. On the other hand, women with similar habits have much more severe TMJ disorders. The findings of this study are in accordance with analogous studies conducted by Okeson et $\mathrm{al}^{[1]}$. His team concluded that adolescent 
females had a higher prevalence of TMD signs and symptoms and carried out oral habits more intensively. Similarly, Gavish et al also carried out a study on oral habits in adolescent females where he found that the prevalence of most oral habits was higher among adolescent females than males ${ }^{[2]}$. Another finding of this study is that a significant difference was found based on individuals who had problems with headaches/migraines in past 6 months.

The number of females having this problem was significantly higher than males.It would seem that these oral habits have a direct relationship to the periodicity of headaches/migraines. In the modern day, different types of chewing gums are being widely used by young subjects.Also the fast paced lifestyle has increased the level of stress that they are burdened with.These factors are leading to development of adverse oral habits.Due to them, the frequency of TMJ disorders has increased amongst this age group. The etiological factors leading to such oral habits need to be recognized and nullified.Only if such steps are taken can these subjects develop into healthy adults.If not, then they will develop complications during later stages of life.

A study carried out by Okeson on the prevalence of oral parafunctions and signs and symptoms of temporomandibular disorders (TMD) in female and male adolescents were compared and their relationship to one another was evaluated. The study consisted of 314 adolescents (136 males and 178 females) and concluded that Adolescent females had a higher prevalence of TMD signs and symptoms and carried out oral habits more intensively. Para functional activity may be another contributing factor in the discrepancies found in the prevalence of signs and symptoms between the sexes. Parafunctional habits and gender may be risk factors of TMD. ${ }^{[1]}$

Another study was carried out by Gavish et al Oral habits and their association with signs and symptoms of temporomandibular disorders in adolescent females. The study consisted of 314 adolescents (136 males and 178 females) and concluded that the prevalence of most oral habits was higher among adolescent females than males. Most examined signs and symptoms of TMD showed a statistically significant higher prevalence among females. ${ }^{[2]}$

Kuttila M, assessed associations between treatment need for temporomandibular disorders (TMD) and age, gender, stress, and diagnostic subgroup were analyzed in an adult Finnish population for 506 subjects. When analyzed separately, the association between TMD treatment need and all the studied factors was statistically significant. This finding is in accordance with earlier results. When the studied factors were included into an explanatory model, however, the picture changed. The logistic regression analysis revealed that diagnostic subgroup was the strongest predictor for the TMD treatment need. Total stress score significantly added to the explanatory power of the model, but age and gender did not. The common place observation that women show more signs and symptoms of TMD seems to be explainable by their higher stress scores and by the type of symptoms. ${ }^{[3]}$

\section{Conclusion}

In the modern day, different types of chewing gums are being widely used by young subjects.Also the fast paced lifestyle has increased the level of stress that they are burdened with.These factors are leading to development of adverse oral habits.Due to them, the frequency of TMJ disorders has increased amongst this age group. The etiological factors leading to such oral habits need to be recognized and nullified.Only if such steps are taken can these subjects develop into healthy adults. If not, then they will develop complications during later stages of life. Further studies with more sample size should be carried out in establishing relationship between headache and tempromandibular joint disorders.

\section{References}

[1] Okesonjp, editor, management of temporomandibular disorders and occlusion. $4^{\text {th }}$ ed. stlouis: cv mosby; 1998 : 149.

[2] Gavish a, oral habits and their association with signs and symptoms of temporomandibular disorders in adolescent females. $\mathrm{J}$ oral rehabil 2000;27:23-32.

[3] Kuttila m,.TMD treatment in relation to age,gender, stress, and diagnostic subgroup. Jorofac pain 1998;12:67-74 .

[4] Ephrain .Oral habits and their association with signs and symptoms of tempromandibular disorders in adolescents: a gender comparison. OOOE.2006;102:482-7.

[5] Samuel F. Dworkin. Research Diagnostic Criteria for Temporomandibular disorders: review, criteria, examinations and specifications, critique 